\title{
Reactivation of Herpes Zoster Keratitis Following Shingrix Vaccine
}

\author{
Tracy J. Lu Christopher N. Ta \\ Department of Ophthalmology, Byers Eye Institute, Stanford University School of Medicine, \\ Palo Alto, CA, USA
}

\section{Keywords}

Shingrix · Shingles · Herpes zoster · Herpes zoster ophthalmicus · Herpes zoster vaccine

Keratitis

\begin{abstract}
We present a case of herpes zoster keratitis reactivation shortly following the Shingrix vaccine. In our patient, reactivation of herpes zoster keratitis occurred a few weeks following the herpes zoster subunit $(\mathrm{HZ} / \mathrm{su})$ vaccine. The development of herpes zoster ophthalmicus following $\mathrm{HZ} / \mathrm{su}$ is exceedingly rare, with only one prior reported case found in the literature. Reporting of this potential correlation is important in understanding the full risks of vaccines and can help elucidate the etiology of such responses.
\end{abstract}

\section{Introduction}

The varicella-zoster virus (VZV) causes varicella, commonly known as chickenpox, which remains dormant in the body after recovery and can reactivate years later to cause herpes zoster, also known as shingles. Herpes zoster can also occur many years following the varicella vaccine, which is made of live-attenuated VZV. The incidence of shingles is relatively common, with one in 3 people estimated to develop shingles in their lifetime, and disproportionately affects older patients ( $>50$ years of age) [1]. Herpes zoster can cause a painful, vesicular rash with sometimes severe sequelae including postherpetic neuralgia and herpes zoster ophthalmicus (HZO), and rarely hearing loss, pneumonia, and even death [1, 2]. HZO occurs due to reactivation of VZV in the ophthalmic branch of the fifth cranial nerve and has a variety of ophthalmic manifestations, including conjunctival injection, chemosis, keratitis, anterior and posterior uveitis, and retinal pathology such as acute retinal necrosis [3]. 
Fig. 1. Baseline photo from December 2018. There is a stromal opacity with cornea thinning, without corneal edema.

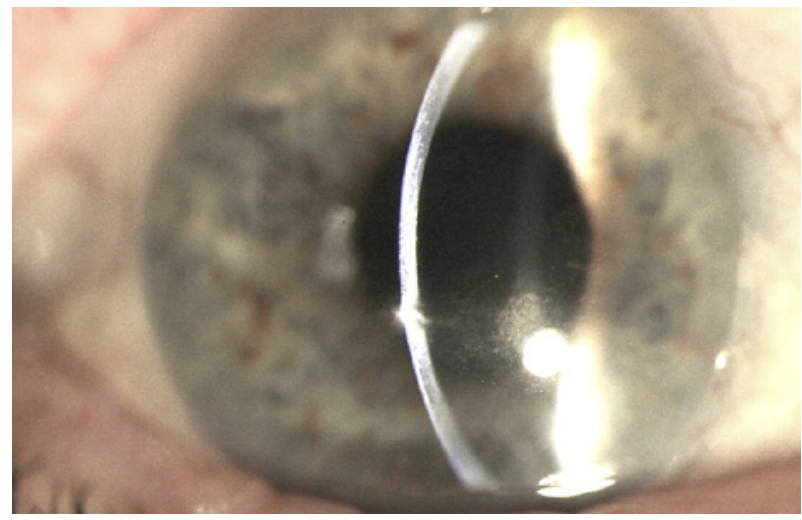

Vaccination is effective in preventing herpes zoster and the associated HZO [4]. The first herpes zoster vaccine, Zostavax, or Zoster Vaccine Live (ZVL) was approved in 2006 and had about a $70 \%$ efficacy rate in patients aged $50-59$ but only $38 \%$ in patients greater than age 70 [5]. A new shingles vaccine, Shingrix, or herpes zoster subunit (HZ/su) vaccine, was approved by the Food and Drug Administration in 2017 and was demonstrated in clinical trials to have an overall efficacy of over $95 \%$ in patients aged 50 or older. HZ/su has now become the preferred herpes zoster vaccine in immunocompetent adults above the age of 50 [6].

There have been uncommon instances in which a patient has developed HZO (either first case or recurrent) after receiving vaccination against herpes zoster [7]. While there have been several reports of uveitis following administration of ZVL or the varicella vaccine for chickenpox (both live-attenuated vaccines) [7], the development of HZO following $\mathrm{HZ} / \mathrm{su}$, which is an inactivated subunit vaccine, is exceedingly rare, with only one prior reported case found in the literature [8]. This paper describes a case of a 75-year-old woman with a history of HZO keratitis (which had been dormant for several years) who experienced a reactivation of keratitis shortly after receiving her first dose of the $\mathrm{HZ} /$ su vaccine.

\section{Case Report}

A 75-year-old woman first developed HZO keratitis in her right eye in 2013. She underwent a prolonged prednisolone taper and was eventually transitioned to fluorometholone twice weekly as a maintenance dose. In 2018, she experienced a reactivation while on fluorometholone, presenting with conjunctival injection, punctate epithelial erosions without dendrites, moderate inferior corneal edema, and stromal haze. She was treated with prednisolone $1 \%$ three times daily to the right eye and oral valacyclovir 1,000 mg three times daily. Two weeks later, her symptoms had completely resolved; the valacyclovir was discontinued, and her prednisolone $1 \%$ was tapered to a maintenance dosage of twice weekly, which she continued to take reliably. She was examined approximately every 3-6 months without evidence of disease reactivation. On baseline examination in November 2020 of her right eye, her visual acuity was $20 / 150$, pinhole to $20 / 70$ with spectacle correction. She had a central stromal opacity with cornea thinning causing irregular astigmatism (shown in Fig. 1). Her pachymetry reading was $424 \mu$. She is pseudophakic with a posterior chamber intraocular lens. The fundus exam was unremarkable. She has glaucoma controlled on latanoprost $0.005 \%$ and timolol $0.5 \%$ in both eyes.

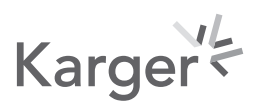


Fig. 2. Slit-lamp photo 1 month after suspected herpes keratitis reactivation was noted. There is inferior corneal edema with corneal folds noted.

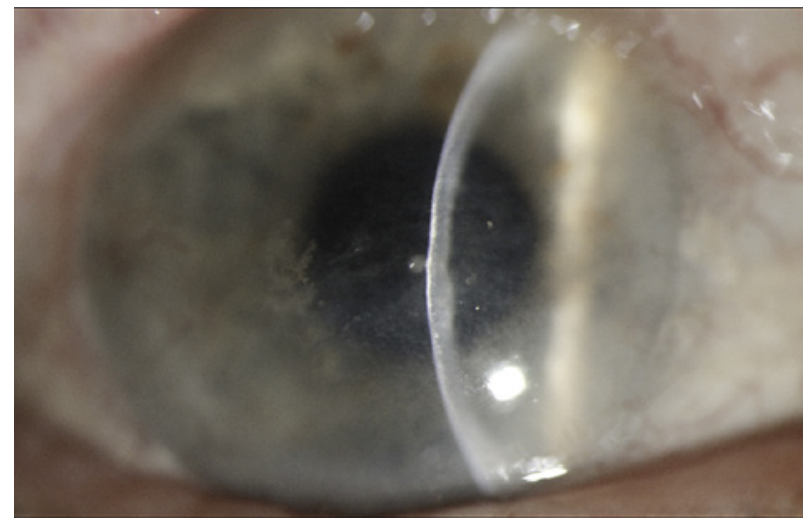

In January 2021, she received her first dose of the HZ/su vaccine. Two and a half weeks later, she was noted by her glaucoma specialist on a routine clinic visit to have a decreased visual acuity of 20/500 with corneal folds in her right eye, which was suspicious for HZO keratitis reactivation. She did not have any recent changes in her glaucoma medications. She was started on oral valacyclovir 1,000 mg twice daily. A month later, the corneal edema persisted (shown in Fig. 2), and her oral valacyclovir was increased to three times daily and prednisolone changed from 1 drop to the right eye twice weekly to three times daily. A week later, her corneal edema was much improved, and the valacyclovir dose was decreased to $1,000 \mathrm{mg}$ daily along with a slow taper of the prednisolone $1 \%$. A month later, her symptoms had completely resolved and her visual acuity returned to a baseline of $20 / 100$ pinhole to 20/80 with no corneal edema on exam. We tapered the prednisolone $1 \%$ to her baseline of twice weekly over a 6 -month period and discontinued the valacyclovir.

\section{Discussion/Conclusion}

The mechanism behind the activation of HZO following the HZ/su vaccine is unknown. For live-attenuated vaccines such as ZVL, it is thought that activation could possibly come from direct inoculation of the attenuated, but still active, virus strain. For inactivated vaccines such as HZ/su, the mechanism is more puzzling; one hypothesis is that one or more adjuvants contained in the vaccines can create an autoinflammatory response, which may either lead to or pose as HZO reactivation [9]. The phenomena of autoinflammatory conditions induced by adjuvants is known collectively as Shoenfeld syndrome and can sometimes include constitutional symptoms such as arthralgia, myalgia, and chronic fatigue (none of which were present in our patient) [10]. The most common causes of Shoenfeld syndrome are aluminum salts which are commonly used in inactivated vaccines [10]. The HZ/su vaccine is not listed as containing aluminum salts, although it does contain a lipid formulation which could be another possible culprit [11]. Another possible explanation is a general upregulated immune response to the vaccine, causing inflammation of the cornea as is observed in herpes zoster keratitis.

In our patient, reactivation of herpes zoster keratitis occurred a few weeks following the $\mathrm{HZ} / \mathrm{su}$ vaccine. Fortunately, she made a complete recovery following treatment with prednisolone 1\% three times a day and Valtrex 1,000 mg three times a day. Her exam returned to baseline with a subsequent slow taper of a topical steroid. The one prior reported case had similarities to our patient: an 89-year-old man with a history of HZO 
keratitis controlled for 1 year on loteprednol etabonate $1 \%$ once daily had developed recurrent keratitis 3 weeks after his first dose of the HZ/su vaccine, with resolution of inflammation following topical application of prednisolone [8]. Post-HZ/su vaccine, HZO reactivation with symptoms other than keratitis has not been reported, raising the possibility that corneal inflammation has a higher propensity for reactivation. Our patient's eye findings only improved with the increased dose of topical steroids and did not respond to systemic antivirals (although initially only on oral valacyclovir 1,000 $\mathrm{mg}$ twice rather than three times daily), suggesting that the keratitis was more likely due to inflammation rather than active viral infection.

Vaccine-associated adverse events such as disease reactivation are uncommon and difficult to prove. Although our patient's keratitis could be coincidental or even related to her glaucoma medications, reporting of possible correlations between $\mathrm{HZ} /$ su vaccine administration and HZO reactivation to public health institutions such as the Vaccine Adverse Event Reporting System, jointly established by the Centers for Disease Control and the Food and Drug Administration, is imperative for accurately understanding the risks associated with certain vaccines and to provide new information that can help elucidate the etiology of these responses.

\section{Statement of Ethics}

Written informed consent was obtained for publication of the details of this medical case and any accompanying images from the patient described in this paper. Ethics approval was not required given that this is a case report, not research, and does not contain any personal health information. This retrospective review of patient data did not require ethical approval in accordance with local/national guidelines.

\section{Conflict of Interest Statement}

The authors have no conflicts of interest to declare.

\section{Funding Sources}

The authors received an unrestricted grant from Research to Prevent Blindness, and the National Eye Institute P30-EY026877.

\section{Author Contributions}

Tracy J. Lu wrote this manuscript and conducted the relevant literature search associated with it. Christopher N. Ta is the senior author who advised and oversaw this case and manuscript.

\section{Data Availability Statement}

All data generated or analyzed during this study are included in this article. Further enquiries can be directed to the corresponding author. 
Lu and Ta: Reactivation of Herpes Zoster Keratitis Following Shingrix Vaccine

\section{References}

1 Cohen JI. Clinical practice: herpes zoster. N Engl J Med. 2013 Jul 18;369(3):255-63.

2 Centers for Disease Control and Prevention (CDC). Shingles (herpes zoster). 2017. Available from: https://www-cdc-gov.laneproxy.stanford.edu/shingles/. (accessed September 18, 2021).

3 Vrcek I, Choudhury E, Durairaj V. Herpes zoster ophthalmicus: a review for the internist. Am J Med. 2017 Jan; 130(1):21-6.

4 Maltz F, Fidler B. Shingrix: a new herpes zoster vaccine. P T. 2019 Jul;44(7):406-33.

5 Dooling KL, Guo A, Patel M, Lee GM, Moore K, Belongia EA, et al. Recommendations of the advisory committee on immunization practices for use of herpes zoster vaccines. MMWR Morb Mortal Wkly Rep. 2018 Jan 26; 67(3):103-108.

6 Lal H, Cunningham AL, Godeaux O, Chlibek R, Diez-Domingo J, Hwang SJ, et al. Efficacy of an adjuvanted herpes zoster subunit vaccine in older adults. N Engl J Med. 2015 May 28;372(22):2087-96.

7 Benage M, Fraunfelder FW. Vaccine-associated uveitis. Mo Med. 2016 Jan-Feb;113(1):48-52.

8 Lehmann A, Matoba A. Reactivation of herpes zoster stromal keratitis after HZ/su adjuvanted herpes zoster subunit vaccine. Ophthalmology. 2018 Nov;125(11):1682.

9 Cunningham ET Jr, Moorthy RS, Moorthy RS, Zierhut M. Vaccine-associated uveitis. Ocul Immunol Inflamm. 2019;27(4):517-20.

10 Watad A, Quaresma M, Brown S, Cohen Tervaert JW, Rodríguez-Pint I, Cervera R, et al. Autoimmune/inflammatory syndrome induced by adjuvants (Shoenfeld's syndrome) - An update. Lupus. 2017 Jun;26(7):675-81.

11 Centers for Disease Control and Prevention [Internet]. Vaccine safety: adjuvants and vaccines. 2021. [cited 2021 Sep 28]. Available from: https://www.cdc.gov/vaccinesafety/concerns/adjuvants.html. 